\title{
The Detection Data of Mammary Carcinoma Processing Method Based on the Improved HHT
}

\author{
http://dx.doi.org/10.3991/ijoe.v9iS6.2847 \\ Zhongling. Han, Zhongming. Han \\ Institute of Information Science and Technology, Shihezi University, Shihezi City, China
}

\begin{abstract}
This paper presents a new algorithm for the analysis of spectral properties of signal of BRATUMASS (Breast tumor microwave sensor system) using the wavelet packet transform (WPT) and the Hilbert-Huang transform (HHT). A wavelet subspace algorithm combined with the empirical mode decomposition (EMD) is introduced to create subdivided intrinsic mode functions (IMFs) and a crosscorrelation analysis is applied to remove pseudo-spectral components. The algorithm proposed by this paper is able to correctly identify spectral patterns of our signal.
\end{abstract}

Index Terms-Hilbert-Huang Transform, Wavelet Packet Transform, BRATUMASS

\section{INTRODUCTION}

Detection of malignant breast tumors at their earliest stage, when they are less than $5 \mathrm{~mm}$ in diameter, remains a challenge. Microwave imaging at frequencies of $1-4 \mathrm{GHz}$ seeks to address the limitations of the existing detection modalities, which include X-ray mammography, ultrasound, and magnetic resonance image (MRI) and all their various imaging modes. The challenge of imaging at these frequencies, however, is resolving tumors when they are $5 \mathrm{~mm}$ or smaller.

The application of microwave imaging (MWI) to breast cancer detection rests on the premise that there exists a detectable contrast between the relative permittivity of malignant breast tissues and normal breast tissues at microwave frequencies. Numerous studies of the electromagnetic dielectric properties of biological tissues at microwave frequencies have shown that the dielectric constant contrast between malignant and benign breast tissues is sufficient to suggest MWI as a useful diagnostic tool for early stage breast cancer detection. These earlier studies suggest the contrast in dielectric constant and conductivity between benign and malignant breast tissues is at least an order of magnitude.

Proceeding on the assumption that MWI techniques will be able to distinguish between malignant and benign breast tissues, many active and passive MWI systems have been proposed. Most systems have focused on active microwave imaging techniques. Each of these systems carries its own set of limitations, which motivates the research effort resulting in the contributions presented in this dissertation.

In active microwave imaging technique, he imaging information of medical diagnosis of mammary tumor in early stage bases on the great different between the dielectric properties of the normal breast tissues and the malignant ones. Therefore, when the electromagnetic wave enters the malignant tumor tissues from the normal mammary tissues, it will interact with the media (for example, absorption, dispersion, emission, etc.), and then the electromagnetic wave transmission path is changed. The electric-field intensity and the magnetic field strength is enhanced in certain regions, while is weakened in other regions. Based on this characteristic, mammary gland tumors can be detection by surveying the electrical field which is produced by the reflection and the scattering generated by near-field microwave. This method can facilitate the early discovery of cancer, early identification of treatment programs and achieve early medical imaging diagnosis of breast cancer. The signals obtained by Breast Tumor Microwave Sensor System (BRATUMASS) are non-linear and non-steady. So the analysis of the detected signals is significantly important for detecting and diagnosing of the early-stage breast tumor. Delays of backscattering in cancerous tissue and the different distance from detecting point to cancerous tissue. The different delays can make the equivalent frequency emerge in anywhere of the effective frequency range of system. Moreover, backscattering occurs in other tissues surface. These aspects increase the frequency component and the difficulties of analysis.

In 1998, Huang et al. introduced a procedure for the spectral analysis of non-stationary nonlinear processes which is now called the Hilbert-Huang transform (HHT). The HHT algorithm decomposes the signal by a procedure called the Empirical Mode Decomposition (EMD), into the so-called Intrinsic Mode Functions (IMF). These IMFs have well defined instantaneous frequencies, and are assumed to represent the intrinsic oscillatory modes embedded in the original signal. The Hilbert spectrum is defined as the sum of the Hilbert spectra obtained for the individual IMFs. This method, unlike conventional Fourier decomposition, can give a very sharp time resolution for the energy-frequency content of the signal, which is one of the main alleged advantages o this method.

Although the HHT may become a promising method to extract the properties of nonlinear and non-stationary signal, like other signal analyses, HHT also suffers from a number of shortcomings. Equation (1) the EMD will generate undesirable IMFs at the low-frequency region that may cause misinterpretation to the result. Equation (2) it depends on the analyzed signal, the first obtained IMF may cover too wide a frequency range that the property of mono-component cannot be achieved. Third, the EMD operation cannot separate signals that contain low-energy components. To solve these shortcomings, an improved HHT is presented here. 


\section{THEORY BASIC}

\section{A. Hilbert-Huang Transform}

Presented by Huang et al. HHT essentially consists of two steps: empirical mode decomposition (EMD) and Hilbert spectral analysis. EMD method will generate a series of intrinsic mode functions (IMFs) and a residue. Expressed in IMFs, they have well-behaved Hilbert transforms, from which the instantaneous frequencies can be calculated.

Given an arbitrary signal $x(t)$, following the EMD method, sifting processes are used to extract the IMFs. The sifting process is shown as in the following equations: (1) and (2)

$$
\begin{aligned}
& m_{1}^{(k+1)}=\frac{u_{1}^{(k+1)}+l_{1}^{(k+1)}}{2} \\
& h_{1}^{(k+1)}=h_{1}^{(k)}-m_{1}^{(k+1)} .
\end{aligned}
$$

where $k=0,1,2 \ldots$ and $h_{1}{ }^{(0)}=x$. One kind of iteration stopping criterion is that the value $S D$ is less than a preselected value, where $S D$ is defined as in

$$
S D=\sum_{i} \frac{\left(h^{(k+1)}\left(t_{i}\right)-h^{(k)}\left(t_{i}\right)\right)^{2}}{\left(h^{(k)}\left(t_{i}\right)\right)^{2}} .
$$

As discussed by Huang, the iteration stopping criterion (3) would be dominated by small local values of $h^{(k)}\left(t_{i}\right)$. By EMD, the original signal $x(t)$ is denoted as in

$$
x(t)=\sum_{i=1}^{n} c_{i}+r_{n}
$$

Having decomposed the signal using the EMD, the Hilbert transform is applied to each IMF. Given a function $x(t)$ its Hilbert transform $y(t)$ is defined as in

$$
y(t)=\frac{1}{\pi} \int_{-\infty}^{\infty} \frac{x(s)}{t-s} d s .
$$

Where the integral is taken as the Cauchy principal value. If $z(t)$ is the analytic signal associated to $x(t)$, we have for all equations about $t$ as in

$$
z(t)=x(t)+j y(t)=A(t) e^{j \theta(t)} .
$$

Where,

$$
\begin{aligned}
& A(t)=\sqrt{x^{2}(t)+y^{2}(t)} . \\
& \theta(t)=\arctan \left(y(t) / x(t) \frac{1}{)} .\right.
\end{aligned}
$$

The instantaneous frequency is now defined as the derivative of the phase function as in

$$
\omega(t)=\frac{d \theta(t)}{d t} .
$$

After decomposition into IMFs, the signal can be represented as in

$$
x(t)=\operatorname{Re}\left[\sum_{i=1}^{n} A_{i}(t) \exp \left(j \int \omega_{i}(t) d t\right)\right] .
$$

Which can be seen as a generalized form of the Fourier decomposition for the function $x(t)$ where both amplitude and frequency are functions of time.

\section{B. The Principle of an Improved HHT Algorithm}

Since the combined IMFs and its residue should be an orthogonal representation of the inspected signal, the relevant IMFs should have strong correlation with the signal, whilst, the irrelevant IMFs should posses weak correlation with the signal. The Wavelet Packet Transform (WPT), due to its well-known properties of being orthogonal, complete, and local, can be a proper preprocessor to separate the inspected signal into various narrow band signals. In order to ensure that you get the IMF meet orthogonal completeness, Peng introduced the WPT and normalized correlation sifting into EMD. This algorithm can offset the defects of EMD and forming a new improved HHT timefrequency analysis method.

\section{(a) Wavelet Packet Transform (WPT)}

For an n-level decomposition, the signal will be decomposed into $2^{n}$ narrow band signals. Note, indifference to the energy content of the component, each component will be decomposed into its bands. That is, even a component has low energy content, unlike the EMD operation, the WPT process will retain its property.

(b) Normalized correlation sifting

The correlation coefficient $\mu$ for each IMF with the inspected signal will be calculated and acted as a criterion for selecting relevant IMFs. For all correlation coefficients $\mu_{i}$, (where $i=1, \ldots, n ; n$ is the number of IMF). The threshold $\lambda$ is set by the ratio of the maximal $\mu_{i}$, which is as in

$$
\lambda=\max \left(\mu_{i}\right) / \eta
$$

Where $\eta$ is a ratio factor. In this study, $\eta=10.0$ is used. Any IMF that has a correlation coefficient equal or higher than $\lambda$ will be retained, whilst, other IMFs will be removed and added to the residue $r$.

The HHT with the WPT as preprocessor plus the IMFs sifting method is here called the improved HHT. Its effectiveness will be verified in the coming section by using both signals generated from a simulated and a real signal of BRATUMASS.

\section{SIGNAL OF BRATUMASS}

BRATUMASS is a time and distance measurement system, which can determine the distance from breast tumor to antenna by obtaining the time delay between directive wave and scattered wave. The position of the breast tumor can locate by the distance.

BRATUMASS consists of RF transceiver module, zero intermediate frequency (zero-IF) mixer. In the transmission, we use slot step frequency modulation method. In the receiver, we sample from zero IF output. In detection process, we arrayed the transceiver (R/T) antenna testing points around the test region and each sounding point of surface-wave (sagittal). There are 12 or 16 detection points of antenna devices, during the real detection process.

In the detecting environment of BRATUMASS, the characteristics and delay of single back wave signal is satisfied Eq. (12) after calculation ${ }^{[9]}$.

$$
\left|\prod\left(\tau_{i}, \omega\right)\right|=2 \pi k_{i} A^{2} \delta\left(\omega-\mu \tau_{i}\right)
$$

where, $\left|\Pi\left(\tau_{i}, \omega\right)\right|$ is the amplitude spectrum of BRAUTMASS signal, $k_{i}$ is the scattering coefficient of target $i, \tau_{i}$ is the delay of back wave, $\mu$ is the FM slope and $A$ is the amplitude of transmitting signal.

The actual detection, the back wave not only exists on the malignant tissue surface, but also on other tissues surface. And relatively speaking, the malignant tissue surface is rougher than normal tissues surface, so the back wave surfaces of malignant tissue are more and relative 
concentration. The makes the time delays, obtained by total back wave signals, large, which are corresponding to more frequency components after mixing. It is very difficult to separate back waves of malignant tissue from total back waves. However, back waves can be separated by using the characteristics of roughly layered distribution of organism tissue structure. That is to say, different tissue layers are corresponding to certain frequency band in effective frequency domain after mixing. We firstly extract layered structure and then separate and distinguish malignant tissue. That can decrease difficulty of distinguish.

The typical actual signal and its FFT, which is shown in Fig.1, obtained from one detecting point, we can only obtain two frequencies: $105 \mathrm{~Hz}$ and $210 \mathrm{~Hz}$. They are additive noise and wave crawled on skin. We couldn't extract any useful signal.

\section{IMPROVED HILBERT-HUANG TRANSFORM OF DETECTION DATA}

All frequency components of back wave are decomposed by using Hilbert-Huang transform (HHT). This method is more suitable for analyzing nonlinear and non-stationary data than Fourier and wavelet method which depend on the priori function.

The high and low frequency components of zero-IF signal are output by HHT decomposition. Fig. 2(a) is the EMD of each frequency component of one detection point output by zero-IF, Fig. 2(b) is the corresponding FFT transformation.

From Fig. 2(b), we can see obviously each frequency component of output zero-IF of each target layer. We take out segregative frequency component from every layer and reconstruct the lesions information, combining with amplitude feature of back signal.

Using the improved HHT algorithm, we can obtain Fig.3. We can extract more fine frequency distribution. Taken out each layer separated frequency component, combined with the signal amplitude of the backscattering characteristics, we can rebuild lesions of information.

From Fig.4, each layer separates out each frequency component in them own frequency band. Synthesized each layer separation results, spectral lines are ascendingly sequenced according its relative distances, which is the distances between the target and the transmit antenna.

As Fig.4 shown, tissue layers are approximate uniformity and continuation in $30 \mathrm{~mm}$. There has a more than $2 \mathrm{~mm}$ discontinuous tissue layer beyond $30 \mathrm{~mm}$, which is roughly fit with anatomical structure of breast. When a detection microwave enter into a breast, it firstly encounters a homogeneous fat layer about $1.5 \mathrm{~cm}(3 \mathrm{~cm} / 2$, microwave have forth and back wave), then it encounters other tissue layers after fat layer.

Fig. 5 is the information of tumor reconstructed from separated frequencies. This image is broadly in line with disease inspection.

\section{CONCLUSION}

Biological information detection data is often nonlinear data, and HHT method for nonlinear and non-stationary signal has a natural advantage. For this kind of signal processing is a very effective analysis method.
We can achieve the effective analysis to the reflected signals by interface of biological tissues using the improved HHT. At the same time, we can distinguish the malignant tissues and the normal tissues by using the relationship of frequency components which are separated and applying proper discrimination model of tissues. However, we don't detailedly discuss the discriminate threshold in the discrimination model of tissues. In fact, this threshold, in the same tissue and in the same microwave condition, is different at largest microwave response. That threshold needs to be further research.
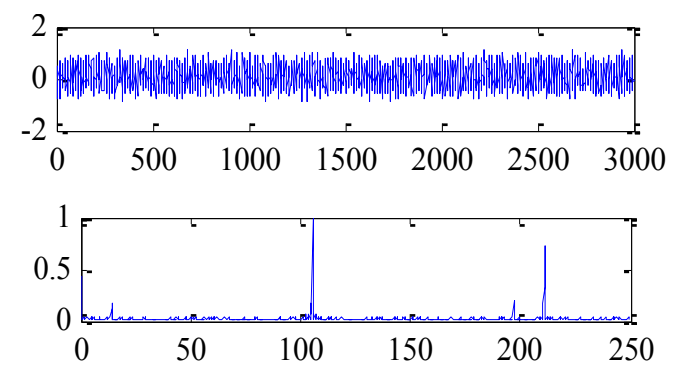

Figure 1. One actual signal and its FFT of BRATUMASS

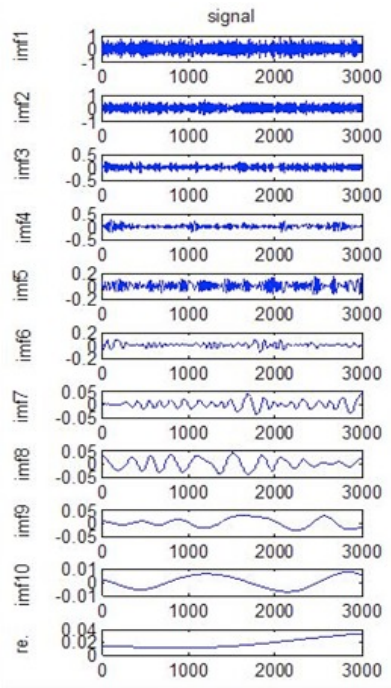

(a)

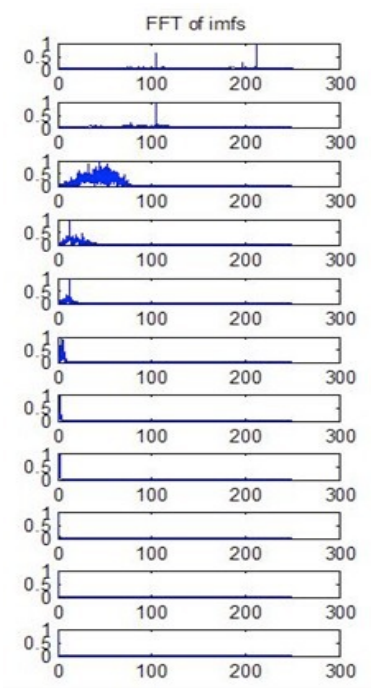

(b)
Figure 2. the EMD of each frequency component of one detection point outputed by zero-IF and the corresponding FFT. (a) the IMFs and the residual of the 9 th detection point, imf1 imf10 is the 10 IMFs, re. is the residual. (b) the Fourier transform of the imf1 imf10; the last one is the Fourier transform of residual.

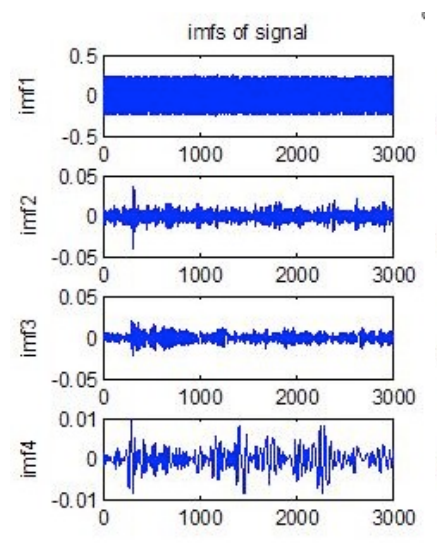

(a)

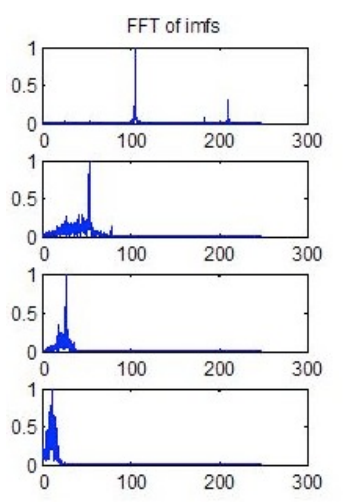

(b)
Figure 3. the improved HHT. (a) the improved HHT of signals of detection point, (b) the FFT of (a) 


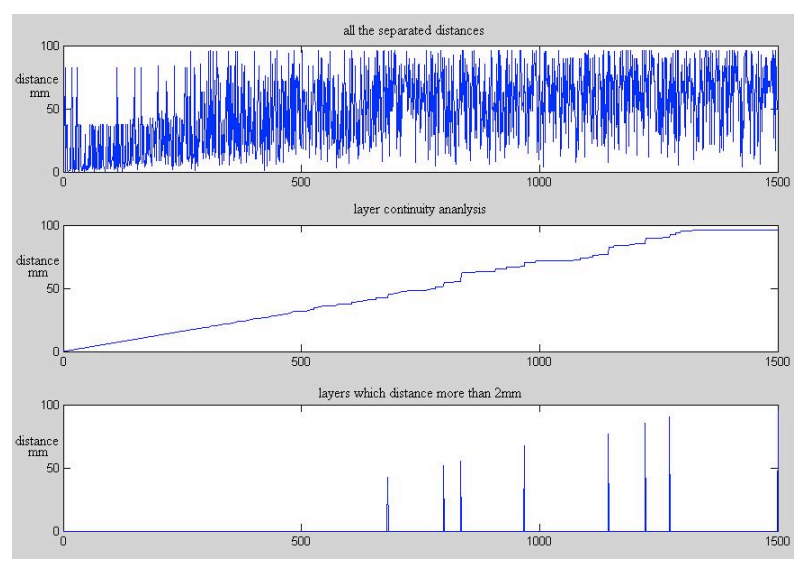

Figure 4. Relative information of tissues layer

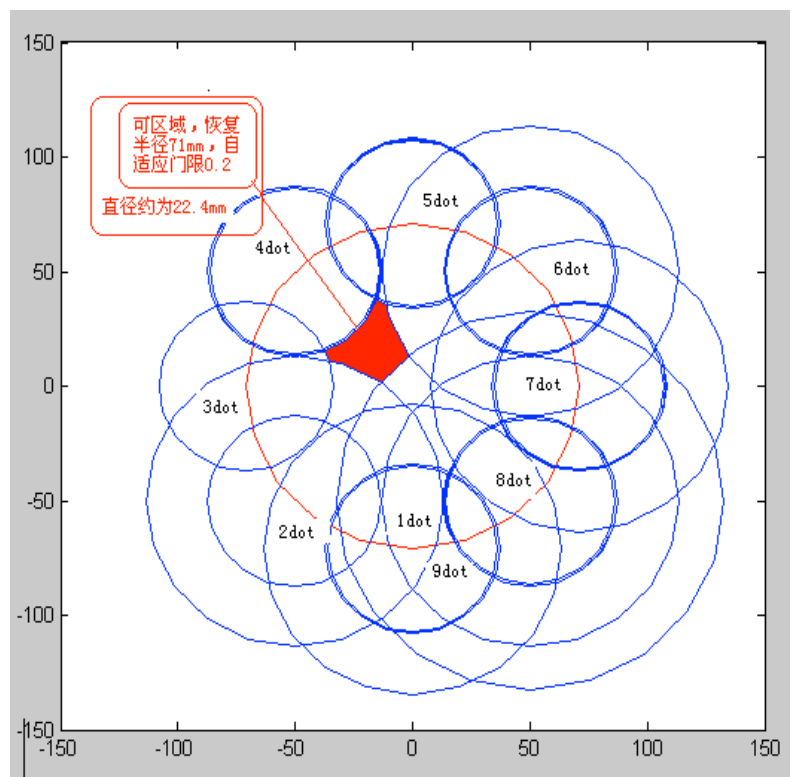

Figure 5. Reconstruction image based on the backscattering frequencies. The red circle as the model reconstruction reference, radius is

$7.1 \mathrm{~cm}$, and the red area is the probable area.

\section{REFERENCE}

[1] C. Gabriel, S. Gabriel, and E. Corthout, "The dielectric properties of biological tissues: I. Literature survey," Physics. Med. Biol, vol. 41, pp. 2231-2249, April1996. http://dx.doi.org/10.1088/00319155/41/11/001

[2] S. Gabriel, R. Lau, and C. Gabriel, "The dielectric properties of biological tissues: II. Measurements in the frequency range $10 \mathrm{~Hz}$ to 20GHz," Physics. Med. Biol, vol.41, pp. 2251-2269, April 1996. http://dx.doi.org/10.1088/0031-9155/41/11/002

[3] C. Gabriel, S. Gabriel, and E. Corthout, "The dielectric properties of biological tissues: III. Parametric models for the dielectric spectrum of tissues," Physics. Med. Biol, vol.41, pp. 2271-2293, April 1996. http://dx.doi.org/10.1088/0031-9155/41/11/003
[4] A. Rosen, M. Stuchly, and A. V. Vorst, "Applications of RF/Microwaves in medicine," IEEE Transactions on Microwave Theory and Techniques, vol. 50, no.3, pp. 963-974, March. 2002.

[5] X. Li, S Davis, S. Hagness, D. van der Weide, and B. V. Veen, "Microwave imaging via space-time beamforming: Experimental inverstigation of tumor detection in multilayer breast phantoms," IEEE Transactions on Microwave Theory and Techniques, vol. 52, no. 8, pp. 1856-1865, August 2004. http://dx.doi.org/10.1109/ TMTT.2004.832686

[6] X. Li, E. Bond, B.V. Veen, and S. Hagness, "An overview of ultra-wideband microwave imaging via space-time beamforming for early-stage breast-cancer detection," IEEE Antennas and Propagation Magazine, vol. 47, no.1, pp.19-34, February 2005. http://dx.doi.org/10.1109/MAP.2005.1436217

[7] J. Bourqui, M. Campbell, J. Sill, M. Shenouda, and E. Fear, "Antenna performance for ultra-wideband microwave imaging," IEEE Radio and Wireless Symposium RWS 2009, pp. 522-525, January 2009.

[8] Huang, N.E., et al., "The empircial mode decomposition and Hilbert spectrum for nonlinear and nonstationary time series analysis," Proceedings of the Royal society of London Series A, vol. 454, pp. 903-995, 1998. http://dx.doi.org/10.1098/rspa.1998. $\underline{0193}$

[9] Peng, Z.K., P.W. Tse, and F.L. Chu, ”An improved Hilbert-Huang transform and its application in vibration signal analysis," Journal of Sound and Vibration, vol. 286, pp. 187-205, August 2005. http://dx.doi.org/10.1016/j.jsv.2004.10.005

[10] Zhifu Tao, Investigation on the Methodologies of Near-Field Microwave Echo Imaging. The Ph.D. Thesis of East China Normal University, 2011.

\section{AUTHORS}

Zhongling. Han comes from Shihezi University in xinjiang province of China, is born in 1980. Now, she is in the study for a doctorate with Communication and information systems professional in East China Normal University. She obtained her master degree with Communication and information systems professional in East China Normal University in 2008. And obtained bachelor degree with Electronic science and technology professional in East China Normal University in 2003. Her major field of study is information processing. Email:hzl_inf@shzu.edu.cn

Zhongming. Han comes from Shihezi University in xinjiang province of China, is born in 1978. Now, he work in Shihezi University. He obtained his master degree with Information management and information systems professional in Tianjin University in 2009. He obtained his bachelor degree with Computer science and technology professional in Shihezi University in 2002. Email:hzm_inf@shzu.edu.cn

This article is an extended and modified version of a paper presented at the 2012 International Conference on Artificial Intelligence and Its Application in Industry Production (AIAIP 2012), held in Wuhan, China in December 2012. Manuscript received 29 May 2013. Published as resubmitted by the authors 26 June 2013. 